\title{
Modification of energy shifts of atoms by the presence of a boundary in a thermal bath and the Casimir-Polder force
}

\author{
Zhiying Zhu and Hongwei $\mathrm{Yu}^{*}$ \\ Department of Physics and Institute of Physics, \\ Hunan Normal University, Changsha, Hunan 410081, China \\ Key Laboratory of Low Dimensional Quantum Structures \\ and Quantum Control of Ministry of Education, \\ Hunan Normal University, Changsha, Hunan 410081, China
}

\begin{abstract}
We study the modification by the presence of a plane wall of energy level shifts of two-level atoms which are in multipolar coupling with quantized electromagnetic fields in a thermal bath in a formalism which separates the contributions of thermal fluctuations and radiation reaction and allows a distinct treatment to atoms in the ground and excited states. The position dependent energy shifts give rise to an induced force acting on the atoms. We are able to identify three different regimes where the force shows distinct features and examine, in all regimes, the behaviors of this force in both the low temperature limit and the high temperature limit for both the ground state and excited state atoms, thus providing some physical insights into the atom-wall interaction at finite temperature. In particular, we show that both the magnitude and the direction of the force acting on an atom may have a clear dependence on atomic the polarization directions. In certain cases, a change of relative ratio of polarizations in different directions may result in a change of direction of the force.
\end{abstract}

PACS numbers: $34.35 .+\mathrm{a}, 12.20 . \mathrm{Ds}, 42.50 . \mathrm{Ct}, 42.50 . \mathrm{Lc}$

\footnotetext{
* Corresponding author
} 


\section{INTRODUCTION}

Quantum fields constrained by the presence of boundaries exhibit many interesting properties because the presence of boundaries disturbs the modes of quantum fields. As a result, fluctuations of quantum fields are altered, giving rise to a lot of novel effects, such as the Casimir [1] and Casimir-Polder forces [2], the light-cone fluctuations when gravity is quantized [3, 4, 5], the Brownian (random) motion of test particles in an electromagnetic vacuum [6, 7, 8, 9, 10, 11], and modifications of radiative properties of atoms in cavities such as the natural lifetimes and energy level shifts which have begun to be demonstrated in experiment [12, 13].

In the present paper, we are particularly interested in the Casmir-Polder force at finite temperature. The Casimir force, which has now been measured with precision [14, 15], is not only fascinating in theoretical research but also increasingly important in technological applications, such as nanophysics [16], chemical identification of surface atoms via atomic force microscope [17] and the construction of novel biomimetic dry adhesives [18].

Casimir-Polder force at zero temperature is usually regarded as a result of the reshaping of vacuum fluctuations induced by the presence of boundaries. In a thorough understanding of the Casimir-Polder force one must also consider the effects of thermal fluctuations in addition to the vacuum ones. It should be pointed out that in the studies of thermal Casimir-Polder force, a majority of researches invokes a macroscopic approach to atoms pioneered by Lifshitz [19, 20, 21] or a linear response theory of atoms [22, 23], although some uses a QED treatment of the atom-field coupling [24, 25, 26].

With an atom-field coupling, the Casimir-Polder force could be understood as a result of the position dependent modification of radiative properties of atoms (the energy level shifts in particular) in the confined space in QED quantitatively. Let us note, however, that a clear and simple physical interpretation does not always emerge from the QED description. One interpretation is that the boundary induced effects on atom's radiative properties may be explained by the modification of vacuum fluctuations of the quantum electromagnetic field [27, 28, 29, 30]. On the other hand, the same effect can also be described as a consequence of 
the reaction of the instantaneous atomic dipole to its own radiation field reflected from the boundary [31, 32, 33, 34]. In fact, it has been shown within QED that the extent to which each mechanism contributes to the total effect can be chosen arbitrarily just by changing the ordering of the atom and field operators in the interaction Hamiltonian [35, 36, 37, 38].

As a result, there exists an indetermination in the separation of effects of vacuum fluctuations and radiation reaction such that distinct contributions of vacuum fluctuations and radiation reaction to the radiative properties of atoms do not possess an independent physical meaning. Therefore, although quantitative results for spontaneous emission and energy level shifts are well-established, the physical interpretations remained controversial until Dalibard, Dupont-Roc and Cohen-Tannoudji(DDC) argued in [39] and [40] that there exists a symmetric operator ordering of atom and field variables where the distinct contributions of vacuum fluctuations and radiation reaction to the rate of change of an atomic observable are separately Hermitian. Recently, this formalism has been employed to study the radiative properties of atoms in various cases [41, 42, 43, 44, 45, 46, 47, 48, 49, 50, 51, 52]. In the present paper, we would like to use the DDC formalism to calculate the modification of energy shifts of atoms by the presence of a boundary in a thermal bath and the forces thus induced between the atom and the boundary. This is not a new problem. However, by using the DDC formalism which separates the contributions of thermal fluctuations (including vacuum fluctuations) and radiation reaction and allows a distinct microscopic treatment to atoms in the ground and excited states, in contrast to the macroscopic approach where atoms are treated as a limiting case of a dielectric, we hope to gain some new physical insight into the atom-wall interactions. Let us note that the same problem at zero temperature has already been discussed in using the DDC formalism [41].

The paper is organized as follows, we give, in Sec. II, a review of the general formalism developed in Ref. [40] and generalized in Ref. [43, 45] to the case of a neutral polarizable two-level atom interacting with quantized electromagnetic fields at finite temperature $T$, and apply it, in Sec. III, to calculate the position dependent energy shifts, which give rise to an induced force acting on the atom. Then we will study, in detail, this force in both the low temperature limit and the high temperature limit for both the ground state and excited 
state atoms. Finally, we will conclude in Sec. IV with a summary of results obtained.

\section{THE THERMAL FLUCTUATIONS AND RADIATION REACTION CONTRI- BUTIONS}

We consider a neutral polarizable two-level atom in interaction with quantized electromagnetic fields in a thermal bath of temperature $T$ in the presence of an infinite conducting plane wall located at $z=0$. We assume that the atom is at rest at a distance, $z$, from the wall, and has stationary states $|-\rangle$ and $|+\rangle$, with energies $-\frac{1}{2} \hbar \omega_{0}$ and $+\frac{1}{2} \hbar \omega_{0}$ and a level spacing $\hbar \omega_{0}$.

To investigate the modification of the atom's energy shifts by the presence of the plane boundary, we will first review the general formalism developed by DDC which allows a distinct separation of the contributions of thermal fluctuations (including zero temperature vacuum fluctuations) and radiation reaction to the energy shifts of atomic levels.

The atom is supposed to interact with the quantized field in the multipolar coupling scheme [53], so that the Hamiltonian that describes the atom-field system with respect to the proper time $\tau$ can be written as

$$
H(\tau)=H_{A}(\tau)+H_{F}(\tau)+H_{I}(\tau)
$$

where

$$
\begin{gathered}
H_{A}(\tau)=\hbar \omega_{0} S_{z}(\tau), \\
H_{F}(\tau)=\sum_{k} \hbar \omega_{\vec{k}} a_{\vec{k}}^{\dagger} a_{\vec{k}} \frac{d t}{d \tau}, \\
H_{I}(\tau)=-\boldsymbol{\mu}(\tau) \cdot \mathbf{E}(x(\tau))=-\boldsymbol{\mu} \cdot \mathbf{E}(x(\tau))\left[S_{+}(\tau)+S_{-}(\tau)\right] .
\end{gathered}
$$

Here $\vec{k}$ denotes the wave vector and polarization of the field modes, $\boldsymbol{\mu}$ the atomic electric dipole moment, and the electric field operator, E, is evaluated along the trajectory $x(\tau)$ of the atom. $S_{z}, S_{+}, S_{-}$are the pseudospin operators of the two-level atom, and $S_{z}(0)=$ $\frac{1}{2}|+\rangle\left\langle+\left|-\frac{1}{2}\right|-\right\rangle\left\langle-\left|, S_{+}(0)=\right|+\right\rangle\left\langle-\left|, S_{-}(0)=\right|-\right\rangle\langle+|$. 
With the Hamiltonian of the system given, we can write down the Heisenberg equations of motion for the dynamical variables of the atom and field from Eq. (1). The solutions of the equations of motion can be split into the two parts: a free part, which is present even in the absence of the coupling, and a source part, which is induced by the interaction of the atom and the field. We assume that the electromagnetic field is in a thermal state with the density matrix $\rho=e^{-\beta H_{F} /(\hbar c)}$, where $\beta=\hbar c /\left(T k_{B}\right)$ is the thermal wavelength, and the atom is initially in the state $|b\rangle . k_{B}$ is the Boltzmann constant. To identify the contributions of thermal fluctuations and radiation reaction to the radiative atomic energy shifts, we choose, following DDC, a symmetric ordering between the atom and the field variables and consider the effects of $E^{f}$ (corresponding to the effect of thermal fluctuations) and $E^{s}$ (corresponding to the effect of radiation reaction) separately in the Heisenberg equations of an arbitrary atomic observable $G$. Following the procedures that have been shown in Refs. [40, 43, 45], we take the thermal average over the electromagnetic field and decompose various rates of $G$ into a Hamiltonian part, describing the energy levels of the atom shifted due to coupling with the electromagnetic field, and a non-Hamiltonian part, describing the effects of relaxation, then in a perturbation treatment up to order $\mu^{2}$, we find

$$
\left\langle\beta\left|\left(\frac{d G(\tau)}{d \tau}\right)_{t f, r r}\right| \beta\right\rangle=\frac{i}{\hbar}\left[H_{t f, r r}^{e f f}(\tau), G(\tau)\right]+\text { non-Hamiltonian terms }
$$

where

$$
\begin{aligned}
& H_{t f}^{e f f}(\tau)=-\frac{i}{2 \hbar} \int_{\tau_{0}}^{\tau} d \tau^{\prime}\left(C_{i j}^{F}\right)_{\beta}\left(x(\tau), x\left(\tau^{\prime}\right)\right)\left[\mu_{i}^{f}(\tau), \mu_{j}^{f}\left(\tau^{\prime}\right)\right], \\
& H_{r r}^{e f f}(\tau)=-\frac{i}{2 \hbar} \int_{\tau_{0}}^{\tau} d \tau^{\prime}\left(\chi_{i j}^{F}\right)_{\beta}\left(x(\tau), x\left(\tau^{\prime}\right)\right)\left\{\mu_{i}^{f}(\tau), \mu_{j}^{f}\left(\tau^{\prime}\right)\right\} .
\end{aligned}
$$

Here $[$,$] and \{$,$\} denote the commutator and anticommutator respectively while subscript "$ $t f$ " stands for thermal fluctuations and "rr" for radiation reaction. The statistical functions $\left(C_{i j}^{F}\right)_{\beta}$ and $\left(\chi_{i j}^{F}\right)_{\beta}$ are defined as

$$
\begin{aligned}
& \left(C_{i j}^{F}\right)_{\beta}\left(x(\tau), x\left(\tau^{\prime}\right)\right)=\frac{1}{2}\left\langle\left\{E_{i}^{f}(x(\tau)), E_{j}^{f}\left(x\left(\tau^{\prime}\right)\right)\right\}\right\rangle_{\beta}, \\
& \left(\chi_{i j}^{F}\right)_{\beta}\left(x(\tau), x\left(\tau^{\prime}\right)\right)=\frac{1}{2}\left\langle\left[E_{i}^{f}(x(\tau)), E_{j}^{f}\left(x\left(\tau^{\prime}\right)\right)\right]\right\rangle_{\beta},
\end{aligned}
$$


which are also called the symmetric correlation function and the linear susceptibility of the field. Taking the expectation value of Eqs. (6) and (77) in the atom's initial state $|b\rangle$, we can obtain the contributions of thermal fluctuations and radiation reaction to the energy shifts of the atom's level $|b\rangle$,

$$
\begin{aligned}
& \left(\delta E_{b}\right)_{t f}=-\frac{i}{\hbar} \int_{\tau_{0}}^{\tau} d \tau^{\prime}\left(C_{i j}^{F}\right)_{\beta}\left(x(\tau), x\left(\tau^{\prime}\right)\right)\left(\chi_{i j}^{A}\right)_{b}\left(\tau, \tau^{\prime}\right), \\
& \left(\delta E_{b}\right)_{r r}=-\frac{i}{\hbar} \int_{\tau_{0}}^{\tau} d \tau^{\prime}\left(\chi_{i j}^{F}\right)_{\beta}\left(x(\tau), x\left(\tau^{\prime}\right)\right)\left(C_{i j}^{A}\right)_{b}\left(\tau, \tau^{\prime}\right),
\end{aligned}
$$

where $\left(C_{i j}^{A}\right)_{b}$ and $\left(\chi_{i j}^{A}\right)_{b}$, the symmetric correlation function and the linear susceptibility of the atom, are defined as

$$
\begin{aligned}
\left(C_{i j}^{A}\right)_{b}\left(\tau, \tau^{\prime}\right) & =\frac{1}{2}\left\langle b\left|\left\{\mu_{i}^{f}(\tau), \mu_{j}^{f}\left(\tau^{\prime}\right)\right\}\right| b\right\rangle, \\
\left(\chi_{i j}^{A}\right)_{b}\left(\tau, \tau^{\prime}\right) & =\frac{1}{2}\left\langle b\left|\left[\mu_{i}^{f}(\tau), \mu_{j}^{f}\left(\tau^{\prime}\right)\right]\right| b\right\rangle,
\end{aligned}
$$

which are characterized by the atom itself. Explicitly, the statistical functions of the atom can be given as

$$
\begin{aligned}
& \left(C_{i j}^{A}\right)_{b}\left(\tau, \tau^{\prime}\right)=\frac{1}{2} \sum_{d}\left[\left\langle b\left|\mu_{i}(0)\right| d\right\rangle\left\langle d\left|\mu_{j}(0)\right| b\right\rangle e^{i \omega_{b d}\left(\tau-\tau^{\prime}\right)}+\left\langle b\left|\mu_{j}(0)\right| d\right\rangle\left\langle d\left|\mu_{i}(0)\right| b\right\rangle e^{-i \omega_{b d}\left(\tau-\tau^{\prime}\right)}\right], \\
& \left(\chi_{i j}^{A}\right)_{b}\left(\tau, \tau^{\prime}\right)=\frac{1}{2} \sum_{d}\left[\left\langle b\left|\mu_{i}(0)\right| d\right\rangle\left\langle d\left|\mu_{j}(0)\right| b\right\rangle e^{i \omega_{b d}\left(\tau-\tau^{\prime}\right)}-\left\langle b\left|\mu_{j}(0)\right| d\right\rangle\left\langle d\left|\mu_{i}(0)\right| b\right\rangle e^{-i \omega_{b d}\left(\tau-\tau^{\prime}\right)}\right],
\end{aligned}
$$

where $\omega_{b d}=\omega_{b}-\omega_{d}$ and the sum extends over a complete set of atomic states. In order to calculate the statistical functions of the electric field, Eqs. (8) and (9), we will use the two point function of the four potential, $A^{\mu}(x)$, at finite temperature, which can be obtained by the method of images both in imaginary-time and in space. In the presence of a boundary, one finds in the Feynman gauge [9],

$$
D_{\beta}^{\mu \nu}\left(x, x^{\prime}\right)=\left\langle A^{\mu}(x) A^{\nu}\left(x^{\prime}\right)\right\rangle_{\beta}=D_{\beta, f r e e}^{\mu \nu}\left(x-x^{\prime}\right)+D_{\beta, \text { bnd }}^{\mu \nu}\left(x, x^{\prime}\right),
$$


where

$$
D_{\beta, \text { free }}^{\mu \nu}\left(x-x^{\prime}\right)=\frac{\hbar}{4 \pi^{2} \varepsilon_{0} c} \sum_{k=-\infty}^{\infty} \frac{\eta^{\mu \nu}}{\left[\left(t c-t^{\prime} c+i k \beta-i \varepsilon\right)^{2}-\left(x-x^{\prime}\right)^{2}-\left(y-y^{\prime}\right)^{2}-\left(z-z^{\prime}\right)^{2}\right]},
$$

is the two point function in the free space and

$$
D_{\beta, b n d}^{\mu \nu}\left(x, x^{\prime}\right)=-\frac{\hbar}{4 \pi^{2} \varepsilon_{0} c} \sum_{k=-\infty}^{\infty} \frac{\eta^{\mu \nu}+2 n^{\mu} n^{\nu}}{\left[\left(t c-t^{\prime} c+i k \beta-i \varepsilon\right)^{2}-\left(x-x^{\prime}\right)^{2}-\left(y-y^{\prime}\right)^{2}-\left(z+z^{\prime}\right)^{2}\right]} .
$$

represents the correction induced by the presence of the boundary. Here $\varepsilon_{0}$ is the vacuum dielectric constant, the subscript " bnd" stands for the part induced by the presence of a boundary, $\varepsilon \rightarrow+0, \eta^{\mu \nu}=\operatorname{diag}(1,-1,-1,-1)$ and the unit normal vector $n^{\mu}=(0,0,0,1)$. Then, we can write the electric field two point function as

$$
\left\langle E_{i}(x(\tau)) E_{j}\left(x\left(\tau^{\prime}\right)\right)\right\rangle_{\beta}=\left\langle E_{i}(x(\tau)) E_{j}\left(x\left(\tau^{\prime}\right)\right)\right\rangle_{\beta, \text { free }}+\left\langle E_{i}(x(\tau)) E_{j}\left(x\left(\tau^{\prime}\right)\right)\right\rangle_{\beta, \text { bnd }},
$$

where

$$
\begin{aligned}
\left\langle E_{i}(x(\tau)) E_{j}\left(x\left(\tau^{\prime}\right)\right)\right\rangle_{\beta, \text { free }}= & \frac{\hbar c}{4 \pi^{2} \varepsilon_{0}} \sum_{k=-\infty}^{\infty}\left(\delta_{i j} \partial_{0} \partial_{0}^{\prime}-\partial_{i} \partial_{j}^{\prime}\right) \\
& \times \frac{1}{\left(x-x^{\prime}\right)^{2}+\left(y-y^{\prime}\right)^{2}+\left(z-z^{\prime}\right)^{2}-\left(t c-t^{\prime} c+i k \beta-i \varepsilon\right)^{2}},
\end{aligned}
$$

and

$$
\begin{aligned}
\left\langle E_{i}(x(\tau)) E_{j}\left(x\left(\tau^{\prime}\right)\right)\right\rangle_{\beta, b n d}= & -\frac{\hbar c}{4 \pi^{2} \varepsilon_{0}} \sum_{k=-\infty}^{\infty}\left[\left(\delta_{i j}-2 n_{i} n_{j}\right) \partial_{0} \partial_{0}^{\prime}-\partial_{i} \partial_{j}^{\prime}\right] \\
& \times \frac{1}{\left(x-x^{\prime}\right)^{2}+\left(y-y^{\prime}\right)^{2}+\left(z+z^{\prime}\right)^{2}-\left(t c-t^{\prime} c+i k \beta-i \varepsilon\right)^{2}} .
\end{aligned}
$$

Here $\partial_{j}^{\prime}$ denotes the differentiation with respect to $x_{j}^{\prime}$. So, the statistical functions of the electric field can be obtained from Eq. (19) as a sum of the free space part and the boundarydependent part. 
Our aim in this paper is to study the modification of atomic energy shifts by the presence of the conducting plane boundary in a thermal bath and the force thus induced on the atom. So in Eqs. (10) and (11), the contributions of thermal fluctuations and radiation reaction to the energy shifts of the atom's level, we are only interested in the boundary-dependent part,

$$
\begin{aligned}
& \left(\delta E_{b}\right)_{t f}^{b n d}=-\frac{i}{\hbar} \int_{\tau_{0}}^{\tau} d \tau^{\prime}\left(C_{i j}^{F}\right)_{\beta, b n d}\left(x(\tau), x\left(\tau^{\prime}\right)\right)\left(\chi_{i j}^{A}\right)_{b}\left(\tau, \tau^{\prime}\right) \\
& \left(\delta E_{b}\right)_{r r}^{b n d}=-\frac{i}{\hbar} \int_{\tau_{0}}^{\tau} d \tau^{\prime}\left(\chi_{i j}^{F}\right)_{\beta, b n d}\left(x(\tau), x\left(\tau^{\prime}\right)\right)\left(C_{i j}^{A}\right)_{b}\left(\tau, \tau^{\prime}\right) .
\end{aligned}
$$

Therefore the modification of energy shifts by the presence of the conducting plane boundary in a thermal bath is given by

$$
\left(\delta E_{b}\right)_{t o t}^{b n d}=\left(\delta E_{b}\right)_{t f}^{b n d}+\left(\delta E_{b}\right)_{r r}^{b n d}
$$

\section{MODIFICATION OF ENERGY SHIFTS BY THE PRESENCE OF A BOUND- ARY IN A THERMAL BATH}

In this section we will apply the formalism developed in the previous section to calculate separately the contributions of the thermal fluctuations and radiation reaction to the energy level shifts of a static two-level atom induced by the presence of a boundary in a thermal bath and the force acting on the atom as a result. We first give the basic results and then proceed to detailed discussions of the behaviors of the position-dependent energy shifts and thus force on the atom both in the low temperature limit (when the wavelength of the thermal photons is much larger than the transition wavelength of the atom) and the high temperature limit (when the wavelength of the thermal photons is much smaller than the transition wavelength of the atom). 


\section{A. Basic results}

For an atom at rest, we can compute the boundary-dependent two point function of the electric field in a thermal bath from the general form Eq. (21) to get

$$
\left\langle E_{i}(x(\tau)) E_{j}\left(x\left(\tau^{\prime}\right)\right)\right\rangle_{\beta, b n d}=-\frac{\hbar c}{\pi^{2} \varepsilon_{0}} \sum_{k=-\infty}^{\infty} \frac{\left(\delta_{i j}-2 n_{i} n_{j}\right)(u c+i k \beta)^{2}+4 z^{2}}{\left[(u c+i k \beta-i \varepsilon)^{2}-4 z^{2}\right]^{3}},
$$

where $u=\tau-\tau^{\prime}$. Consequently, the boundary-dependent statistical functions of the electric field can be expressed as

$$
\begin{aligned}
& \left(C_{i j}^{F}\right)_{\beta, b n d}\left(x(\tau), x\left(\tau^{\prime}\right)\right)=-\frac{\hbar c}{2 \pi^{2} \varepsilon_{0}} \sum_{k=-\infty}^{\infty}\left(\frac{\left(\delta_{i j}-2 n_{i} n_{j}\right)(u c+i k \beta)^{2}+4 z^{2}}{\left[(u c+i k \beta-i \varepsilon)^{2}-4 z^{2}\right]^{3}}\right. \\
& \left.+\frac{\left(\delta_{i j}-2 n_{i} n_{j}\right)(u c+i k \beta)^{2}+4 z^{2}}{\left[(u c+i k \beta+i \varepsilon)^{2}-4 z^{2}\right]^{3}}\right), \\
& \left(\chi_{i j}^{F}\right)_{\beta, b n d}\left(x(\tau), x\left(\tau^{\prime}\right)\right)=-\frac{\hbar c}{2 \pi^{2} \varepsilon_{0}}\left(\frac{\left(\delta_{i j}-2 n_{i} n_{j}\right) u^{2} c^{2}+4 z^{2}}{\left[(u c-i \varepsilon)^{2}-4 z^{2}\right]^{3}}-\frac{\left(\delta_{i j}-2 n_{i} n_{j}\right) u^{2} c^{2}+4 z^{2}}{\left[(u c+i \varepsilon)^{2}-4 z^{2}\right]^{3}}\right) .
\end{aligned}
$$

The linear susceptibility of the electromagnetic field, Eq. (27), is independent of the temperature. Let us note that only the diagonal components $(i=j=x, y, z)$ of the statistical functions are nonzero, and the components parallel to the conducting plane, i.e., the $x x$ and

yy components, are equal. Substituting the nonzero statistical functions of the atom and the electric field into the general formulas (22) and (23), we can obtain the boundary-dependent contributions of thermal fluctuations and radiation reaction to the energy shifts of atomic levels,

$$
\begin{aligned}
\left(\delta E_{b}\right)_{t f}^{b n d}= & \frac{i c}{4 \pi^{2} \varepsilon_{0}} \sum_{k=-\infty}^{\infty} \sum_{j d}\left|\left\langle b\left|\mu_{j}(0)\right| d\right\rangle\right|^{2} \int_{0}^{\infty} d u\left(e^{i \omega_{b d} u}-e^{-i \omega_{b d} u}\right) \\
& \times\left(\frac{\left(1-2 n_{j} n_{j}\right)(u c+i k \beta)^{2}+4 z^{2}}{\left[(u c+i k \beta-i \varepsilon)^{2}-4 z^{2}\right]^{3}}+\frac{\left(1-2 n_{j} n_{j}\right)(u c+i k \beta)^{2}+4 z^{2}}{\left[(u c+i k \beta+i \varepsilon)^{2}-4 z^{2}\right]^{3}}\right),
\end{aligned}
$$

and

$$
\begin{aligned}
\left(\delta E_{b}\right)_{r r}^{b n d}= & \frac{i c}{4 \pi^{2} \varepsilon_{0}} \sum_{j d}\left|\left\langle b\left|\mu_{j}(0)\right| d\right\rangle\right|^{2} \int_{0}^{\infty} d u\left(e^{i \omega_{b d} u}+e^{-i \omega_{b d} u}\right) \\
& \times\left(\frac{\left(1-2 n_{j} n_{j}\right) u^{2} c^{2}+4 z^{2}}{\left[(u c-i \varepsilon)^{2}-4 z^{2}\right]^{3}}-\frac{\left(1-2 n_{j} n_{j}\right) u^{2} c^{2}+4 z^{2}}{\left[(u c+i \varepsilon)^{2}-4 z^{2}\right]^{3}}\right) .
\end{aligned}
$$


Here we have extend the range of integration to infinity for sufficiently long times $\tau-\tau_{0}$. The integration can be evaluated using contour integrals with the help of the residue theorem. With a definition of the atomic static scalar polarizability

$$
\alpha_{0}=\sum_{j} \alpha_{j}=\sum_{j d} \frac{2\left|\left\langle b\left|\mu_{j}(0)\right| d\right\rangle\right|^{2}}{3 \omega_{0} \hbar},
$$

the results can be cast into

$$
\left(\delta E_{+}\right)_{t f}^{b n d}=\frac{3 \hbar \omega_{0} \alpha_{j}}{128 \pi \varepsilon_{0}}\left[\left(1+\frac{2}{e^{\beta \omega_{0} / c}-1}\right) f_{j}\left(\omega_{0}, z\right)-g_{j}\left(\omega_{0}, z, \beta\right)\right]
$$

for the contribution of thermal fluctuations to the energy level shift of the excited state,

$$
\left(\delta E_{-}\right)_{t f}^{b n d}=-\frac{3 \hbar \omega_{0} \alpha_{j}}{128 \pi \varepsilon_{0}}\left[\left(1+\frac{2}{e^{\beta \omega_{0} / c}-1}\right) f_{j}\left(\omega_{0}, z\right)-g_{j}\left(\omega_{0}, z, \beta\right)\right],
$$

for that of the ground state, and

$$
\left(\delta E_{+}\right)_{r r}^{b n d}=\left(\delta E_{-}\right)_{r r}^{b n d}=\frac{3 \hbar \omega_{0} \alpha_{j}}{128 \pi \varepsilon_{0}} f_{j}\left(\omega_{0}, z\right)
$$

for the contribution of radiation reaction to the energy shifts of both the ground and excited states. Here we have defined

$$
\begin{gathered}
f_{x}\left(\omega_{0}, z\right)=f_{y}\left(\omega_{0}, z\right)=\frac{4 z^{2} \omega_{0}^{2}-c^{2}}{z^{3} c^{2}} \cos \left(2 z \omega_{0} / c\right)-\frac{2 \omega_{0}}{z^{2} c} \sin \left(2 z \omega_{0} / c\right), \\
f_{z}\left(\omega_{0}, z\right)=-\frac{2}{z^{3}} \cos \left(2 z \omega_{0} / c\right)-\frac{4 \omega_{0}}{z^{2} c} \sin \left(2 z \omega_{0} / c\right), \\
g_{x}\left(\omega_{0}, z, \beta\right)=g_{y}\left(\omega_{0}, z, \beta\right)=\frac{64 c}{\pi} \sum_{k=-\infty}^{\infty} \int_{0}^{\infty} d u \frac{(u c+k \beta)^{2}-4 z^{2}}{\left[(u c+k \beta)^{2}+4 z^{2}\right]^{3}} e^{-\omega_{0} u}, \\
g_{z}\left(\omega_{0}, z, \beta\right)=-\frac{64 c}{\pi} \sum_{k=-\infty}^{\infty} \int_{0}^{\infty} d u \frac{1}{\left[(u c+k \beta)^{2}+4 z^{2}\right]^{2}} e^{-\omega_{0} u},
\end{gathered}
$$

and summation over repeated indexes is implied. We note that the contribution of the radiation reaction to the energy shifts, Eq. (33), does not depend on the temperature and the contribution of fluctuations of fields to the energy level shift of the ground state is just 
opposite to that of the excited state. Adding up the contributions of thermal fluctuations and radiation reaction, we arrive at the position-dependent energy shift,

$$
\left(\delta E_{+}\right)_{t o t}^{b n d}=\frac{3 \hbar \omega_{0} \alpha_{j}}{128 \pi \varepsilon_{0}}\left[\left(2+\frac{2}{e^{\beta \omega_{0} / c}-1}\right) f_{j}\left(\omega_{0}, z\right)-g_{j}\left(\omega_{0}, z, \beta\right)\right]
$$

for the excited state, and

$$
\left(\delta E_{-}\right)_{t o t}^{b n d}=-\frac{3 \hbar \omega_{0} \alpha_{j}}{128 \pi \varepsilon_{0}}\left[\frac{2}{e^{\beta \omega_{0} / c}-1} f_{j}\left(\omega_{0}, z\right)-g_{j}\left(\omega_{0}, z, \beta\right)\right]
$$

for the ground state. Since the energy shifts depend on the distance $z$ between the atom and the conducting plane, the atom feels a force near the wall. In what follows, we examine in detail the behaviors of this force in various circumstances, considering separately the ground and excited atoms.

\section{B. Low temperature limit}

Let us now discuss the case when the wavelength of thermal photons is much larger than the transition wavelength of the atom, $\lambda_{0}$,

$$
\beta \gg \lambda_{0}
$$

where $\lambda_{0}=c / \omega_{0}$. This condition, which makes transitions from the ground state to the excited state virtually impossible, is very well satisfied at the usual room temperature. As a matter of fact, the first optical resonance of $\mathrm{Rb}$ atoms is as high as $1.8 \times 10^{4} \mathrm{~K}$. We will analyze how the interaction energy or the force acting on the atom behaves as the distance varies in three different regimes: the short distance, where the distance $z$ is much smaller than the transition wavelength of the atom $\left(z \ll \lambda_{0}\right)$, the intermediate distance, where the distance $z$ is much larger than the transition wavelength of the atom but much smaller than the thermal wavelength $\left(\lambda_{0} \ll z \ll \beta\right)$, and the long distance, where the distance $z$ is much larger than the thermal wavelength $\left(z \gg \beta \gg \lambda_{0}\right)$. For these three distinct regimes, we will discuss the force on a ground state atom and an excited one separately. 


\section{Ground state atom}

Let us start with the short distance regime, i.e., when $z \ll \lambda_{0}$. In this case, thermal fluctuations and radiation reaction contributions to the boundary-dependent energy shift become approximately,

$$
\begin{aligned}
\left(\delta E_{-}\right)_{t f}^{b n d} \approx-\frac{\hbar}{4 \pi \varepsilon_{0}}[ & -\frac{3 \omega_{0}^{2} \alpha_{z}}{4 \pi c z^{2}}-\frac{\omega_{0}^{4}}{c^{3}}\left(\alpha_{x}+\alpha_{y}-\alpha_{z}\right) \log \left(z \omega_{0} / c\right) \\
& \left.-\left(\frac{2 \pi^{3} c}{15 \beta^{4}}+\frac{16 \pi^{5} c^{3}}{63 \omega_{0}^{2} \beta^{6}}\right)\left(\alpha_{x}+\alpha_{y}-\alpha_{z}\right)+\frac{32 \pi^{5} c z^{2}}{315 \beta^{6}}\left(2 \alpha_{x}+2 \alpha_{y}-\alpha_{z}\right)\right],
\end{aligned}
$$

and

$$
\left(\delta E_{-}\right)_{r r}^{b n d} \approx-\frac{\hbar}{4 \pi \varepsilon_{0}} \frac{3 \omega_{0}}{32 z^{3}}\left(\alpha_{x}+\alpha_{y}+2 \alpha_{z}\right) .
$$

Here the contribution of radiation reaction is much larger than that of thermal fluctuations, and plays the dominant role in the total energy shift. Consider for example, a Rb atom $\left(\omega_{0}=2.37 \times 10^{15} s^{-1}\right)$ placed 1 nanometer from the conducting boundary at room temperature $(T=300 K)$, then the contribution of radiation reaction is about 200 times as large as that of thermal fluctuations. The closer the atom is to the boundary, the larger is this relative ratio.

Since we are interested in the force on the atom, we will drop the $z$-independent terms. Then we find by adding up the two contributions

$$
\begin{aligned}
\left(\delta E_{-}\right)_{t o t}^{b n d} \approx-\frac{\hbar}{4 \pi \varepsilon_{0}}[ & \frac{3 \omega_{0}}{32 z^{3}}\left(\alpha_{x}+\alpha_{y}+2 \alpha_{z}\right)-\frac{3 \omega_{0}^{2} \alpha_{z}}{4 \pi c z^{2}}-\frac{\omega_{0}^{4}}{c^{3}}\left(\alpha_{x}+\alpha_{y}\right) \log \left(z \omega_{0} / c\right) \\
& \left.+\frac{32 \pi^{5} c z^{2}}{315 \beta^{6}}\left(2 \alpha_{x}+2 \alpha_{y}-\alpha_{z}\right)\right] .
\end{aligned}
$$

The first (leading) term on the righthand side of Eq. (43) is just the atom-wall interaction

energy at zero temperature in the short distance limit obtained in Ref. 2] (an extra factor of $\hbar /\left(4 \pi \varepsilon_{0}\right)$ is caused by the SI units used in the present paper) while the last term gives leading thermal corrections. The leading position dependent energy shift leads to the van der Waals force and the calculations above show clearly that the source of this force is purely 
radiation reaction. The energy shift given in Eq. (43) induces an attractive force between a ground state atom and the plane wall. It is also interesting to note that the atom's polarization in the longitudinal and transverse directions are weighted differently in terms of their contribution to the force. For a ground state atom polarized isotropically the position dependent interaction energy shift reads

$$
\left(\delta E_{-}\right)_{\text {tot }}^{\text {bnd }} \approx-\frac{\hbar}{4 \pi \varepsilon_{0}}\left(\frac{\omega_{0} \alpha_{0}}{8 z^{3}}+\frac{32 \pi^{5} c \alpha_{0} z^{2}}{315 \beta^{6}}\right),
$$

where the first term is just the usual van der Waals result. One should bear in mind, however, that the polarization in different directions contributes differently in the van der Waals force even for isotropically polarized atoms. In fact, the van der Waals force on a ground state atom polarizable only in $z$ direction would be twice as that polarizable only in the parallel directions. This is one of the new understandings we gain by not treating the atom as a limiting case of a macroscopic dielectric.

Now let us turn to intermediate distance regime, i.e., when $\lambda_{0} \ll z \ll \beta$. Here one finds

$$
\begin{aligned}
\left(\delta E_{-}\right)_{t f}^{b n d} \approx-\frac{\hbar}{4 \pi \varepsilon_{0}}[ & \left(\frac{3 \omega_{0}^{3}\left(\alpha_{x}+\alpha_{y}\right)}{8 c^{2} z}-\frac{3 \omega_{0}}{32 z^{3}}\left(\alpha_{x}+\alpha_{y}+2 \alpha_{z}\right)\right) \cos \left(2 z \omega_{0} / c\right) \\
& -\frac{3 \omega_{0}^{2}}{16 c z^{2}}\left(\alpha_{x}+\alpha_{y}+2 \alpha_{z}\right) \sin \left(2 z \omega_{0} / c\right)+\frac{3 c}{8 \pi z^{4}}\left(\alpha_{x}+\alpha_{y}+\alpha_{z}\right) \\
& \left.+\frac{32 \pi^{5} c z^{2}}{315 \beta^{6}}\left(2 \alpha_{x}+2 \alpha_{y}-\alpha_{z}\right)\right]
\end{aligned}
$$

and

$$
\begin{aligned}
\left(\delta E_{-}\right)_{r r}^{b n d} \approx \frac{\hbar}{4 \pi \varepsilon_{0}}[ & \left(\frac{3 \omega_{0}^{3}\left(\alpha_{x}+\alpha_{y}\right)}{8 c^{2} z}-\frac{3 \omega_{0}}{32 z^{3}}\left(\alpha_{x}+\alpha_{y}+2 \alpha_{z}\right)\right) \cos \left(2 z \omega_{0} / c\right) \\
& \left.-\frac{3 \omega_{0}^{2}}{16 c z^{2}}\left(\alpha_{x}+\alpha_{y}+2 \alpha_{z}\right) \sin \left(2 z \omega_{0} / c\right)\right] .
\end{aligned}
$$

Both the contributions of the thermal fluctuations and the radiation reaction have terms which are oscillating functions of $z$. However, these oscillating components cancel out when added up, leading to

$$
\left(\delta E_{-}\right)_{t o t}^{b n d} \approx-\frac{\hbar}{4 \pi \varepsilon_{0}}\left[\frac{3 c}{8 \pi z^{4}}\left(\alpha_{x}+\alpha_{y}+\alpha_{z}\right)+\frac{32 \pi^{5} c z^{2}}{315 \beta^{6}}\left(2 \alpha_{x}+2 \alpha_{y}-\alpha_{z}\right)\right],
$$

where the first term is completely coincident with the zero temperature Casimir-Polder interaction energy given in Ref. [2] and the atom's polarization in different direction contributes 
equally. The second gives the thermal correction which is equal to that in the short distance regime and it however depends on the atom's polarization direction. One sees that the Casimir-Polder force is a result of a residue energy shift due to vacuum fluctuations after cancelations of competing oscillating terms from thermal fluctuations and radiation reaction. This demonstrates that the Casimir-Polder force originates from a net result of vacuum fluctuations at zero temperature and radiation reaction. Here there is no clear domination of one effect over the other as is in the short distance regime. In fact, the two effects are now comparable and opposing each other, and the net effect is much smaller than one either of them. We demonstrate this feature graphically in Fig. 1(a) and Fig. 1(b), For an isotropically polarized atom, we obtain

$$
\left(\delta E_{-}\right)_{t o t}^{b n d} \approx-\frac{\hbar}{4 \pi \varepsilon_{0}}\left(\frac{3 c \alpha_{0}}{8 \pi z^{4}}+\frac{32 \pi^{5} c \alpha_{0} z^{2}}{315 \beta^{6}}\right)
$$

which is always negative and the force on the ground state atom is again attractive in the intermediate distance regime.

Finally, for the long distance regime, i.e., when $z \gg \beta \gg \lambda_{0}$, one has

$$
\begin{aligned}
\left(\delta E_{-}\right)_{t f}^{b n d} \approx-\frac{\hbar}{4 \pi \varepsilon_{0}}[ & \left(\frac{3 \omega_{0}^{3}\left(\alpha_{x}+\alpha_{y}\right)}{8 c^{2} z}-\frac{3 \omega_{0}}{32 z^{3}}\left(\alpha_{x}+\alpha_{y}+2 \alpha_{z}\right)\right) \cos \left(2 z \omega_{0} / c\right) \\
& \left.-\frac{3 \omega_{0}^{2}}{16 c z^{2}}\left(\alpha_{x}+\alpha_{y}+2 \alpha_{z}\right) \sin \left(2 z \omega_{0} / c\right)+\frac{3 c}{16 z^{3} \beta}\left(\alpha_{x}+\alpha_{y}+2 \alpha_{z}\right)\right]
\end{aligned}
$$

and

$$
\begin{aligned}
\left(\delta E_{-}\right)_{r r}^{b n d} \approx \frac{\hbar}{4 \pi \varepsilon_{0}}[ & \left(\frac{3 \omega_{0}^{3}\left(\alpha_{x}+\alpha_{y}\right)}{8 c^{2} z}-\frac{3 \omega_{0}}{32 z^{3}}\left(\alpha_{x}+\alpha_{y}+2 \alpha_{z}\right)\right) \cos \left(2 z \omega_{0} / c\right) \\
& \left.-\frac{3 \omega_{0}^{2}}{16 c z^{2}}\left(\alpha_{x}+\alpha_{y}+2 \alpha_{z}\right) \sin \left(2 z \omega_{0} / c\right)\right] .
\end{aligned}
$$

Similar to the intermediate distance regime $\left(\lambda_{0} \ll z \ll \beta\right)$, the oscillating terms in the contributions of thermal fluctuations and radiation reaction balance each other. So, again, in the present case, there is no clear domination of one effect over the other. However, the position-dependent energy shift of the ground state now has the form

$$
\left(\delta E_{-}\right)_{\text {tot }}^{\text {bnd }} \approx-\frac{\hbar}{4 \pi \varepsilon_{0}} \frac{3 c}{16 z^{3} \beta}\left(\alpha_{x}+\alpha_{y}+2 \alpha_{z}\right)=-\frac{1}{4 \pi \varepsilon_{0}} \frac{3 k_{B} T}{16 z^{3}}\left(\alpha_{x}+\alpha_{y}+2 \alpha_{z}\right) .
$$


This is linear in the temperature and depends on the polarization direction of the atom too. In the present case, the contributions from vacuum fluctuations are negligible as compared to that of thermal fluctuations in clear contrast to the intermediate regime. Therefore, in the low temperature limit $\left(\beta \gg \lambda_{0}\right)$, only when the distance between the ground state atom and the conducting boundary is much larger than the thermal wavelength $(z \gg \beta)$, do the thermal fluctuations become the dominating source of the position-dependent energy shifts and thus of the force acting on the atom. It is particularly interesting to note that the force acting on a ground state atom which is transversely polarized is twice as much as that of the atom which is longitudinally polarized. For an isotropically polarized ground state atom the total contributions

$$
\left(\delta E_{-}\right)_{t o t}^{b n d} \approx-\frac{1}{4 \pi \varepsilon_{0}} \frac{\alpha_{0} k_{B} T}{4 z^{3}} .
$$

This is in agreement with the Lifshitz's result [19], which was obtained in a macroscopic approach to the problem where the force between two dielectric semi-infinite spaces at finite temperature was considered and the force on an atom is obtained as a limiting case when one dielectric is sufficiently rarefied. Note that the force is again attractive.

\section{Excited state atom}

After having analyzed the force on an atom in the ground state in the low temperature limit, let us now discuss what happens if the atom is in the excited state. One notes, from Eqs. (31)-(33), that the contributions of thermal fluctuations to the energy shifts for the ground and excited state atoms are of the same magnitude but opposite signs, while that of the radiation reaction are equal. Since radiation reaction is temperature independent, the thermal corrections to energy shift of the excited state come solely from thermal fluctuations and thus will be just opposite to that of the ground state. As a result, in the short distance regime where the contributions from radiation reaction dominate over thermal fluctuations (and vacuum fluctuations), one finds

$$
\left(\delta E_{+}\right)_{t o t}^{b n d} \approx-\frac{\hbar}{4 \pi \varepsilon_{0}}\left[\frac{3 \omega_{0}}{32 z^{3}}\left(\alpha_{x}+\alpha_{y}+2 \alpha_{z}\right)-\frac{32 \pi^{5} c z^{2}}{315 \beta^{6}}\left(2 \alpha_{x}+2 \alpha_{y}-\alpha_{z}\right)\right]
$$


which agrees with the result for the ground state atom in the leading order(refer to Eq. (43)). Therefore, in the low temperature and short distance limit, the force acting on the atom reduces to the Van der Waals result for both the ground and excited in the leading order plus a small thermal corrections which differs in sign. The net force is attractive. It is worth pointing out here that the force on excited atoms, in general, may not be necessarily the gradient of a potential. However, in the intermediate distance regime, where radiation reaction no longer dominates, the total energy level shift deviates considerably from that of the ground state as a result of the fact that the oscillating terms from thermal fluctuations and radiation reaction do not cancel as they do in the case of the ground state atom. The result is

$$
\begin{aligned}
\left(\delta E_{+}\right)_{\text {tot }}^{\text {bnd }} \approx \frac{\hbar}{4 \pi \varepsilon_{0}}[ & \left(\frac{3 \omega_{0}^{3}\left(\alpha_{x}+\alpha_{y}\right)}{4 c^{2} z}-\frac{3 \omega_{0}}{16 z^{3}}\left(\alpha_{x}+\alpha_{y}+2 \alpha_{z}\right)\right) \cos \left(2 z \omega_{0} / c\right) \\
& -\frac{3 \omega_{0}^{2}}{8 c z^{2}}\left(\alpha_{x}+\alpha_{y}+2 \alpha_{z}\right) \sin \left(2 z \omega_{0} / c\right)+\frac{3 c}{8 \pi z^{4}}\left(\alpha_{x}+\alpha_{y}+\alpha_{z}\right) \\
& \left.+\frac{32 \pi^{5} c z^{2}}{315 \beta^{6}}\left(2 \alpha_{x}+2 \alpha_{y}-\alpha_{z}\right)\right] .
\end{aligned}
$$

Amplitude of the oscillating terms is much larger than the Casimir-Polder term which behaves like $1 / z^{4}$ and consequently, for a given atom, the position-dependent energy shift of the excited state may be much larger than that of the ground state depending on the distance $z$ of the atom from the boundary. The thermal corrections are very small compared with the vacuum fluctuation contributions. For an isotropically polarized atom, we can write Eq. (54) as

$\left(\delta E_{+}\right)_{t o t}^{b n d} \approx \frac{\hbar}{4 \pi \varepsilon_{0}}\left[\left(\frac{\omega_{0}^{3} \alpha_{0}}{2 c^{2} z}-\frac{\omega_{0} \alpha_{0}}{4 z^{3}}\right) \cos \left(2 z \omega_{0} / c\right)-\frac{\omega_{0}^{2} \alpha_{0}}{2 c z^{2}} \sin \left(2 z \omega_{0} / c\right)+\frac{3 c \alpha_{0}}{8 \pi z^{4}}+\frac{32 \pi^{5} c \alpha_{0} z^{2}}{315 \beta^{6}}\right]$,

which may be positive or negative depending on the distance $z$ so that the force acting on the excited state atom may be attractive or repulsive and even be zero. This is quite different from the case of a ground state atom, where the position-dependent energy shift is always negative and the force is attractive. It is interesting to note that, for a given atom, there exist certain values of the distance $z$ such that the oscillatory terms sum up to zero and 
when that happens the position-dependent energy shift becomes

$$
\left(\delta E_{+}\right)_{t o t}^{b n d} \approx \frac{\hbar}{4 \pi \varepsilon_{0}}\left(\frac{3 c \alpha_{0}}{8 \pi z^{4}}+\frac{32 \pi^{5} c \alpha_{0} z^{2}}{315 \beta^{6}}\right)
$$

which leads to a force on the excited atom equal in magnitude to that on ground state atom but opposite in direction. It is remarkable that whenever the oscillatory terms do not sum up to zero, the force on the excited atom is generally much stronger than the usual CasimirPolder force on the ground state atom and alternates between attractive and repulsive as the distance varies. The total energy shift for an excited state is plotted in Fig. 1(c) along with Fig. 1 to show this.

Now it is time to look at the long distance regime. It is easy to show that the energy shift can be approximated as

$$
\begin{aligned}
\left(\delta E_{+}\right)_{t o t}^{b n d} \approx \frac{\hbar}{4 \pi \varepsilon_{0}}[ & \left(\frac{3 \omega_{0}^{3}\left(\alpha_{x}+\alpha_{y}\right)}{4 c^{2} z}-\frac{3 \omega_{0}}{16 z^{3}}\left(\alpha_{x}+\alpha_{y}+2 \alpha_{z}\right)\right) \cos \left(2 z \omega_{0} / c\right) \\
& \left.-\frac{3 \omega_{0}^{2}}{8 c z^{2}}\left(\alpha_{x}+\alpha_{y}+2 \alpha_{z}\right) \sin \left(2 z \omega_{0} / c\right)+\frac{3 c}{16 z^{3} \beta}\left(\alpha_{x}+\alpha_{y}+2 \alpha_{z}\right)\right]
\end{aligned}
$$

Here the oscillating terms arising from vacuum fluctuations are the same as in the intermediate distance regime but the thermal correction term $\left(1 / z^{3} \beta\right)$ overtakes the Casimir-Polder one $\left(1 / z^{4}\right)$. A comparison with Eq. (51) shows that, although the thermal effects plays a major role in the position-dependent energy shift for the ground state, it is generally not the case for the excited state because of the presence of oscillating terms. In fact, the thermal correction term is of higher order when compared with the oscillating terms, unless the atom is such placed that the oscillatory terms sum up to zero. Therefore, the net contributions of vacuum fluctuations and radiation reaction in general dominate even in the long distance regime if the atom is in the excited state, as opposed to the ground state where thermal effects dominate in the long distance regime. For an isotropically polarized atom,

$$
\left(\delta E_{+}\right)_{t o t}^{b n d} \approx \frac{\hbar}{4 \pi \varepsilon_{0}}\left[\left(\frac{\omega_{0}^{3} \alpha_{0}}{2 c^{2} z}-\frac{\omega_{0} \alpha_{0}}{4 z^{3}}\right) \cos \left(2 z \omega_{0} / c\right)-\frac{\omega_{0}^{2} \alpha_{0}}{2 c z^{2}} \sin \left(2 z \omega_{0} / c\right)+\frac{c \alpha_{0}}{4 z^{3} \beta}\right],
$$

which again can be positive or negative depending on the position of the atom.

The above discussions reveal that the force acting on the excited atom is definitely attractive only in the short distance regime and can be either attractive or repulsive in the 
intermediate and long distance regime. This is in a clear contrast to the ground state atom where the force is attractive in all regimes. When the oscillatory terms sum up to zero, we have

$$
\left(\delta E_{+}\right)_{t o t}^{b n d} \approx \frac{\hbar}{4 \pi \varepsilon_{0}} \frac{c \alpha_{0}}{4 z^{3} \beta}=\frac{1}{4 \pi \varepsilon_{0}} \frac{\alpha_{0} k_{B} T}{4 z^{3}}
$$

leading to a force which is equal to the Lifshitz result in magnitude but opposite in direction.

\section{High temperature limit}

Now we turn our attention to the high temperature limit, where we assume the wavelength of the thermal photons to be much smaller than the transition wavelength of the atom, i.e.,

$$
\beta \ll \lambda_{0}
$$

which can also be written as $\beta \omega_{0} / c \ll 1$. Then Eqs. (31) and (32), for the contributions of the thermal fluctuations to the position-dependent energy shifts, can be approximated as

$$
\left(\delta E_{-}\right)_{t f}^{b n d}=-\left(\delta E_{+}\right)_{t f}^{b n d} \approx-\frac{3 \hbar \omega_{0} \alpha_{j}}{128 \pi \varepsilon_{0}}\left[\frac{2 c}{\beta \omega_{0}} f_{j}\left(\omega_{0}, z\right)-g_{j}\left(\omega_{0}, z, \beta\right)\right]
$$

As in the low temperature limit, we will separately examine the position-dependent energy shifts and thus the force acting on the atom for both the ground and excited states in three different distance regimes.

We first consider the case when the atom is placed so close to the boundary that the distance $z$ is much smaller than the thermal wavelength $\left(z \ll \beta \ll \lambda_{0}\right)$. It follows that

contributions of thermal fluctuations and radiation reaction to the energy level shifts are now respectively,

$$
\left(\delta E_{-}\right)_{t f}^{b n d}=-\left(\delta E_{+}\right)_{t f}^{b n d} \approx \frac{\hbar}{4 \pi \varepsilon_{0}} \frac{3 c}{16 \beta z^{3}}\left(\alpha_{x}+\alpha_{y}+2 \alpha_{z}\right)
$$

and

$$
\left(\delta E_{-}\right)_{r r}^{b n d}=\left(\delta E_{+}\right)_{r r}^{b n d} \approx-\frac{\hbar}{4 \pi \varepsilon_{0}} \frac{3 \omega_{0}}{32 z^{3}}\left(\alpha_{x}+\alpha_{y}+2 \alpha_{z}\right)
$$


Here the contributions of thermal fluctuations (62) are much larger than that of the radiation reaction (63) and play the dominating role in the position-dependent energy shifts for both the ground and excited state. It is interesting to note that in the high temperature limit, even when the distance $z$ is much smaller than the wavelength of thermal photons, the thermal effects dominate over zero-point contributions. This is in contrast to the low temperature limit where thermal effects dominate only in the long distance regime when the distance $z$ is much larger than the wavelength of thermal photons. For an isotropically polarized atom, we obtain

$$
\left(\delta E_{-}\right)_{t o t}^{b n d}=-\left(\delta E_{+}\right)_{t o t}^{b n d} \approx \frac{\hbar}{4 \pi \varepsilon_{0}} \frac{c \alpha_{0}}{4 \beta z^{3}}=\frac{1}{4 \pi \varepsilon_{0}} \frac{\alpha_{0} k_{B} T}{4 z^{3}},
$$

which is linear in temperature, and gives a positive position-dependent energy shift for a ground state atom and a negative one for an excited state. This means that the force acting on an atom is repulsive if the atom is in the ground state and attractive if otherwise. Notice that the force on a ground state atom in the present case is different from the Lifshitz's result in the low temperature and long distance limit in direction although it is equal in magnitude.

If the distance $z$ is much larger than the thermal wavelength but much smaller than the transition wavelength, i.e., $\beta \ll z \ll \lambda_{0}$, then we find

$$
\left(\delta E_{-}\right)_{t f}^{b n d}=-\left(\delta E_{+}\right)_{t f}^{b n d} \approx-\frac{\hbar}{4 \pi \varepsilon_{0}}\left[\frac{3 \omega_{0}^{2}}{8 c \beta z}\left(\alpha_{x}+\alpha_{y}-2 \alpha_{z}\right)-\frac{9 \omega_{0}^{4} z}{8 c^{3} \beta}\left(\alpha_{x}+\alpha_{y}-\frac{2}{3} \alpha_{z}\right)\right],
$$

and

$$
\left(\delta E_{-}\right)_{r r}^{b n d}=\left(\delta E_{+}\right)_{r r}^{b n d} \approx-\frac{\hbar}{4 \pi \varepsilon_{0}} \frac{3 \omega_{0}}{32 z^{3}}\left(\alpha_{x}+\alpha_{y}+2 \alpha_{z}\right) .
$$

For the total position-dependent energy shifts, we can obtain

$$
\left(\delta E_{-}\right)_{t o t}^{b n d} \approx-\frac{\hbar}{4 \pi \varepsilon_{0}}\left[\frac{3 \omega_{0}^{2}}{8 c \beta z}\left(\alpha_{x}+\alpha_{y}-2 \alpha_{z}\right)-\frac{9 \omega_{0}^{4} z}{8 c^{3} \beta}\left(\alpha_{x}+\alpha_{y}-\frac{2}{3} \alpha_{z}\right)+\frac{3 \omega_{0}}{32 z^{3}}\left(\alpha_{x}+\alpha_{y}+2 \alpha_{z}\right)\right]
$$

for the ground state, and

$$
\left(\delta E_{+}\right)_{t o t}^{b n d} \approx \frac{\hbar}{4 \pi \varepsilon_{0}}\left[\frac{3 \omega_{0}^{2}}{8 c \beta z}\left(\alpha_{x}+\alpha_{y}-2 \alpha_{z}\right)-\frac{9 \omega_{0}^{4} z}{8 c^{3} \beta}\left(\alpha_{x}+\alpha_{y}-\frac{2}{3} \alpha_{z}\right)-\frac{3 \omega_{0}}{32 z^{3}}\left(\alpha_{x}+\alpha_{y}+2 \alpha_{z}\right)\right] .
$$


for the excited state. Here there is again no strong domination of one effect over the other. However, the above results show that now whether the force acting on the atom is attractive or repulsive depends crucially on the polarizations of the atom. For an example, a change of relative ratio of polarizations in different directions may result in a change of direction of the force. We note that for an isotropically polarized atom, the first term in both Eq. (667) and (68) is zero, so

$$
\left(\delta E_{-}\right)_{t o t}^{b n d} \approx \frac{\hbar}{4 \pi \varepsilon_{0}}\left(\frac{\omega_{0}^{4} \alpha_{0} z}{2 c^{3} \beta}-\frac{\omega_{0} \alpha_{0}}{8 z^{3}}\right)
$$

and

$$
\left(\delta E_{+}\right)_{t o t}^{b n d} \approx-\frac{\hbar}{4 \pi \varepsilon_{0}}\left(\frac{\omega_{0}^{4} \alpha_{0} z}{2 c^{3} \beta}+\frac{\omega_{0} \alpha_{0}}{8 z^{3}}\right)
$$

Here for an excited state atom, the position-dependent energy shift is always negative and induces an attractive force, but for a ground state atom, it is determined by the magnitude of the distance $z$ and the wavelength of the thermal photon. The crucial dependence of the direction of the force acting the atom on its polarization in this case is an interesting feature of atom-wall interactions revealed in the present paper.

Thirdly, for the long distance limit such that the distance $z$ is much bigger than the transition wavelength $\left(z \gg \lambda_{0} \gg \beta\right)$, the contributions of thermal fluctuations and radiation reaction can be written as

$$
\begin{aligned}
\left(\delta E_{-}\right)_{t f}^{b n d}=-\left(\delta E_{+}\right)_{t f}^{b n d} \approx-\frac{\hbar}{4 \pi \varepsilon_{0}} & {\left[\left(\frac{3 \omega_{0}^{2}\left(\alpha_{x}+\alpha_{y}\right)}{4 c \beta z}-\frac{3 c}{16 \beta z^{3}}\left(\alpha_{x}+\alpha_{y}+2 \alpha_{z}\right)\right) \cos \left(2 z \omega_{0} / c\right)\right.} \\
& \left.\frac{3 \omega_{0}}{8 \beta z^{2}}\left(\alpha_{x}+\alpha_{y}+2 \alpha_{z}\right) \sin \left(2 z \omega_{0} / c\right)+\frac{3 c}{16 \beta z^{3}}\left(\alpha_{x}+\alpha_{y}+2 \alpha_{z}\right)\right]
\end{aligned}
$$

and

$$
\begin{aligned}
\left(\delta E_{-}\right)_{r r}^{b n d}=\left(\delta E_{+}\right)_{r r}^{b n d} \approx \frac{\hbar}{4 \pi \varepsilon_{0}}[ & \left(\frac{3 \omega_{0}^{3}\left(\alpha_{x}+\alpha_{y}\right)}{8 c^{2} z}-\frac{3 \omega_{0}}{32 z^{3}}\left(\alpha_{x}+\alpha_{y}+2 \alpha_{z}\right)\right) \cos \left(2 z \omega_{0} / c\right) \\
& \left.-\frac{3 \omega_{0}^{2}}{16 c z^{2}}\left(\alpha_{x}+\alpha_{y}+2 \alpha_{z}\right) \sin \left(2 z \omega_{0} / c\right)\right]
\end{aligned}
$$

Clearly, in the present case, the contribution of radiation reaction is much smaller than that of thermal fluctuations since $\beta \omega_{0} / c \ll 1$ and thus the vacuum fluctuation effect dominates. 
So the position-dependent energy shifts of both the ground and excited states are dominated by the contribution of thermal fluctuations. Assuming isotropic polarization, we arrive at

$$
\begin{aligned}
\left(\delta E_{-}\right)_{t o t}^{b n d}=-\left(\delta E_{+}\right)_{t o t}^{b n d} & \approx-\frac{\hbar}{4 \pi \varepsilon_{0}}\left[\left(\frac{\omega_{0}^{2} \alpha_{0}}{2 c \beta z}-\frac{c \alpha_{0}}{4 \beta z^{3}}\right) \cos \left(2 z \omega_{0} / c\right)-\frac{\omega_{0} \alpha_{0}}{2 \beta z^{2}} \sin \left(2 z \omega_{0} / c\right)+\frac{c \alpha_{0}}{4 \beta z^{3}}\right] \\
& \approx-\frac{\hbar}{4 \pi \varepsilon_{0}}\left[\frac{\omega_{0}^{2} \alpha_{0}}{2 c \beta z} \cos \left(2 z \omega_{0} / c\right)-\frac{\omega_{0} \alpha_{0}}{2 \beta z^{2}} \sin \left(2 z \omega_{0} / c\right)+\frac{c \alpha_{0}}{4 \beta z^{3}}\right]
\end{aligned}
$$

which has both terms of oscillating function of distance $z$ and a Lifshitz-like term. If the atom is so placed that the oscillating part is equal to zero, the position-dependent energy shift of the ground state reduces to the Lifshitz's result.

In all the discussions above, we have only studied energy level shifts of an atom in its ground and excited state separately. An interesting issue yet to be addressed is the thermal average of energy level shifts of an atom in the equilibrium with thermal photons. This is particularly desirable in the high temperature limit, since now the ground state atoms have a high possibility to absorb thermal photons to transit to the excited state. Taking into account the fermionic nature of the two-level atom, which can be seen by noting that the atomic raising and lowering operators obey the anticommutation relation, $\left\{S_{+}, S_{-}\right\}=1$, we can calculate the thermal average of contributions of the thermal fluctuations and radiation reaction to the position-dependent energy shifts as follows,

$$
\begin{aligned}
(\overline{\delta E})_{t f}^{b n d} & =\frac{1}{1+e^{-\omega_{0} \beta / c}}\left(\delta E_{-}\right)_{t f}^{b n d}+\left(1-\frac{1}{1+e^{-\omega_{0} \beta / c}}\right)\left(\delta E_{+}\right)_{t f}^{b n d} \\
& =\frac{3 \hbar \omega_{0} \alpha_{j}}{128 \pi \varepsilon_{0}}\left[-f_{j}\left(\omega_{0}, z\right)+\frac{1-e^{-\omega_{0} \beta / c}}{1+e^{-\omega_{0} \beta / c}} g_{j}\left(\omega_{0}, z, \beta\right)\right]
\end{aligned}
$$

and

$$
\begin{aligned}
(\overline{\delta E})_{r r}^{b n d} & =\frac{1}{1+e^{-\omega_{0} \beta / c}}\left(\delta E_{-}\right)_{r r}^{b n d}+\left(1-\frac{1}{1+e^{-\omega_{0} \beta / c}}\right)\left(\delta E_{+}\right)_{r r}^{b n d} \\
& =\frac{3 \hbar \omega_{0} \alpha_{j}}{128 \pi \varepsilon_{0}} f_{j}\left(\omega_{0}, z\right) .
\end{aligned}
$$

When the temperature is low, the thermal average shift is essentially the shift of the ground state as expected, and significant deviations only occur when temperature is high. So, in 
what follows, we will examine in detail the average energy shifts in the three distinct distance regimes in the high temperature limit.

First, the short distance regime, where the distance $z$ is assumed to be much smaller than the thermal wavelength, i.e., $z \ll \beta \ll \lambda_{0}$. Now the contributions of thermal fluctuations and radiation reaction to the average energy shifts can be approximated as

$$
(\overline{\delta E})_{t f}^{b n d} \approx \frac{\hbar}{4 \pi \varepsilon_{0}}\left(\frac{3 \omega_{0}}{32 z^{3}}-\frac{3 \omega_{0}^{2} \beta}{64 c z^{3}}\right)\left(\alpha_{x}+\alpha_{y}+2 \alpha_{z}\right)
$$

and

$$
(\overline{\delta E})_{r r}^{b n d} \approx-\frac{\hbar}{4 \pi \varepsilon_{0}} \frac{3 \omega_{0}}{32 z^{3}}\left(\alpha_{x}+\alpha_{y}+2 \alpha_{z}\right)
$$

Here one can see that the contribution of temperature-independent vacuum fluctuations to the average energy shift cancels that of the radiation reaction when added up, leaving a correction due to thermal fluctuations as the dominant term in the average energy shift. So

$$
(\overline{\delta E})_{t o t}^{b n d} \approx-\frac{\hbar}{4 \pi \varepsilon_{0}} \frac{3 \omega_{0}^{2} \beta}{64 c z^{3}}\left(\alpha_{x}+\alpha_{y}+2 \alpha_{z}\right)=-\frac{1}{4 \pi \varepsilon_{0}} \frac{3 \hbar^{2} \omega_{0}^{2}}{64 k_{B} T z^{3}}\left(\alpha_{x}+\alpha_{y}+2 \alpha_{z}\right),
$$

This means that the average energy shift decreases as the temperature increases in the short distance regime $\left(z \ll \beta \ll \lambda_{0}\right)$. For isotropic polarization, Eq. (78) becomes

$$
(\overline{\delta E})_{\text {tot }}^{\text {bnd }} \approx-\frac{1}{4 \pi \varepsilon_{0}} \frac{\hbar^{2} \omega_{0}^{2} \alpha_{0}}{16 k_{B} T z^{3}} .
$$

This is always negative and leads to an attractive average force on the atom.

Second, we consider the intermediate distance regime, where the distance $z$ is much larger than the thermal wavelength but much smaller than the transition wavelength of the atom, i.e., $\beta \ll z \ll \lambda_{0}$. Now one finds

$$
\begin{gathered}
(\overline{\delta E})_{t f}^{\text {bnd }} \approx-\frac{\hbar}{4 \pi \varepsilon_{0}}\left[\frac{3 \omega_{0}^{3}}{16 c^{2} z}\left(\alpha_{x}+\alpha_{y}-2 \alpha_{z}\right)+\frac{3 \omega_{0}^{2}}{4 \pi c z^{2}} \alpha_{z}-\frac{\omega_{0}^{3} \beta^{2}}{128 c^{2} z^{3}}\left(\alpha_{x}+\alpha_{y}+2 \alpha_{z}\right)\right] \\
(\overline{\delta E})_{r r}^{b n d} \approx-\frac{\hbar}{4 \pi \varepsilon_{0}} \frac{3 \omega_{0}}{32 z^{3}}\left(\alpha_{x}+\alpha_{y}+2 \alpha_{z}\right)
\end{gathered}
$$

so the average energy shift is given by

$$
(\overline{\delta E})_{t o t}^{b n d} \approx-\frac{\hbar}{4 \pi \varepsilon_{0}}\left(\frac{3 \omega_{0}}{32 z^{3}}-\frac{\omega_{0}^{3} \beta^{2}}{128 c^{2} z^{3}}\right)\left(\alpha_{x}+\alpha_{y}+2 \alpha_{z}\right) .
$$


Here the average energy shift is caused mainly by the radiation reaction and the thermal correction due to thermal fluctuations is quadratic in $\beta$ in contrast to the linear dependence in the short distance regime. For an isotropically polarized atom, Eq. (82) reduces to

$$
(\overline{\delta E})_{t o t}^{b n d} \approx-\frac{\hbar}{4 \pi \varepsilon_{0}}\left(\frac{\omega_{0} \alpha_{0}}{8 z^{3}}-\frac{\omega_{0}^{3} \beta^{2} \alpha_{0}}{96 c^{2} z^{3}}\right)
$$

where the first term is just the Van der Waals result.

Finally, we can estimate the contributions of thermal fluctuations and radiation reaction to the average energy shift, in the long distance regime $\left(z \gg \lambda_{0} \gg \beta\right)$, to get

$$
\begin{aligned}
(\overline{\delta E})_{t f}^{b n d} \approx-\frac{\hbar}{4 \pi \varepsilon_{0}}[ & \left(\frac{3 \omega_{0}^{3}\left(\alpha_{x}+\alpha_{y}\right)}{8 c^{2} z}-\frac{3 \omega_{0}}{32 z^{3}}\left(\alpha_{x}+\alpha_{y}+2 \alpha_{z}\right)\right) \cos \left(2 z \omega_{0} / c\right) \\
& \left.-\frac{3 \omega_{0}^{2}}{16 c z^{2}}\left(\alpha_{x}+\alpha_{y}+2 \alpha_{z}\right) \sin \left(2 z \omega_{0} / c\right)+\left(\frac{3 \omega_{0}}{32 z^{3}}-\frac{\omega_{0}^{3} \beta^{2}}{128 c^{2} z^{3}}\right)\left(\alpha_{x}+\alpha_{y}+2 \alpha_{z}\right)\right]
\end{aligned}
$$

and

$$
\begin{aligned}
(\overline{\delta E})_{r r}^{b n d} \approx \frac{\hbar}{4 \pi \varepsilon_{0}}[ & \left(\frac{3 \omega_{0}^{3}\left(\alpha_{x}+\alpha_{y}\right)}{8 c^{2} z}-\frac{3 \omega_{0}}{32 z^{3}}\left(\alpha_{x}+\alpha_{y}+2 \alpha_{z}\right)\right) \cos \left(2 z \omega_{0} / c\right) \\
& \left.-\frac{3 \omega_{0}^{2}}{16 c z^{2}}\left(\alpha_{x}+\alpha_{y}+2 \alpha_{z}\right) \sin \left(2 z \omega_{0} / c\right)\right] .
\end{aligned}
$$

Noting that the oscillating terms in the contributions of thermal fluctuations are canceled out by that of the radiation reaction in the calculation of the total shift, we obtain

$$
(\overline{\delta E})_{t o t}^{b n d} \approx-\frac{\hbar}{4 \pi \varepsilon_{0}}\left(\frac{3 \omega_{0}}{32 z^{3}}-\frac{\omega_{0}^{3} \beta^{2}}{128 c^{2} z^{3}}\right)\left(\alpha_{x}+\alpha_{y}+2 \alpha_{z}\right)
$$

This coincides with Eq. (82) for the case of $\beta \ll z \ll \lambda_{0}$ in mathematical appearance but differs in physical origin. The reason is that here the average energy shift comes mainly from the contribution of the thermal fluctuations while in the intermediate distance regime the radiation reaction plays the major role.

\section{CONCLUSION}

Using the DDC formalism which separates the contributions of thermal fluctuations and radiation reaction and allows a distinct microscopic treatment to atoms in the ground and 
excited states, in contrast to the macroscopic approach where atoms are treated as a limiting case of a dielectric, we have investigated the modification by the presence of a plane wall of energy level shifts of two-level atoms which are in multipolar coupling with quantized electromagnetic fields at the finite temperature. The position dependent energy shifts give rise to an induced force acting on the atoms. We have examined the behaviors of this force in both the low temperature limit and the high temperature limit for both the ground state and excited state atoms.

In the low temperature limit where the wavelength of thermal photons is assumed to be much larger than the transition wavelength of the atom, our calculations reproduce, for a ground state atom, the van der Waals force at short distance regime, the Casmir-Polder force in the intermediate regime and the Lifshitz result in the long distance regime, and show that the force is attractive in all three regimes. However if the atom is in the excited state, the atom-wall force agrees with that in the ground state in the leading order only in the short distance limit. In the intermediate distance regime the force on the excited state atoms is generally strong than the Casimir-Polder force on the ground state atoms and alternates between attractive and repulsive as the atom's distance from the wall varies. In the long distance regime, the main source of the force is the net contributions of competing vacuum fluctuation and radiation reaction and the thermal correction term is in general of higher order. This is in clear contrast to the ground state atom where the thermal effect (the Lifshitz term) dominates. Furthermore, the force can again be attractive and repulsive depending on the atom's distance.

In the high temperature limit where the wavelength of thermal photons is assumed to be much smaller than the transition wavelength of the atom, we find that the thermal effects dominate over zero-point contributions even in the short distance limit where the distance is small as compared to the transition wavelength and the force is linear in temperature in the leading order in all three regimes. In the short distance regime the force on an excited atom is attractive while that on a ground state atom is repulsive. In the intermediate distance, the direction of the force acting on the atoms depends crucially the polarization. Only in the long distance regime does the force have oscillatory part, leading to a force which would 
either be attractive or repulsive.

We also have calculated the thermal average of energy level shifts for an ensemble of atoms in equilibrium with the thermal photons in the high temperature limit. We find that the average force is attractive and decreases as $1 / T$ as temperature $T$ grows in the short distance regime. However, both in the intermediate and long distance regimes, the average force coincides with the van de Waals force in the leading order, which is curiously independent of the temperature. One should note, however, that although the force has the same form in two different regimes, the physical origin is quite different, with the force in the intermediate regime being generated by radiation reaction and that in long distance by thermal fluctuations.

By not treating atoms as a limiting case of a macroscopic dielectric, we are able to reveal the influence of different atomic polarizations on the energy level shifts and thus the force acting on the atoms. Interestingly, we find that both the magnitude and the direction of the force acting on an atom have a clear dependence on the atomic polarization directions. In certain cases, a change of relative ratio of polarizations in different directions may result in a change of direction of the force.

Finally, as far as the separation of effects of fluctuations and radiation reaction in the DDC prescription itself is concerned, we only find a clear domination of one over the other in short distance regime in the low temperature limit where radiation reaction effect dominates and in both the short and long distance regimes in the high temperature limit where fluctuations effect does. An interesting issue for future discussions is the non-equilibrium situation where the atom and field have different temperatures and the two terms can thus be varied independently. In fact, in this case, the contribution of radiation reaction (atom fluctuations) would only depend on the atomic temperature and the thermal fluctuation contribution (field fluctuations) would only depend on the field temperature. We hope to return to this issue in the future. 


\section{Acknowledgments}

This work was supported in part by the National Natural Science Foundation of China under Grants No. 10575035, 10775050, the SRFDP under Grant No. 20070542002, and the Hunan Provincial Innovation Foundation for Postgraduate.

[1] H. B. G. Casimir, Proc. K. Ned. Akad. Wet. 51, 793 (1948).

[2] H. B. G. Casimir and D. Polder, Phys. Rev. 73, 360 (1948).

[3] H. Yu and L. H. Ford, Phys. Rev. D 60, 084023 (1999).

[4] H. Yu and L. H. Ford, Phys. Lett. B 496, 107 (2000); gr-qc/0004063.

[5] H. Yu and P.X. Wu, Phys. Rev. D 68, 084019 (2003).

[6] H. Yu and L. H. Ford, Phys. Rev. D 70, 065009 (2004).

[7] H. Yu and J. Chen, Phys. Rev. D 70, 125006 (2004).

[8] M. Tan and H. Yu, Chin. Phys. Lett. 22, 2165 (2005).

[9] H. Yu, J. Chen, and P. Wu, J. High Energy Phys. 02058 (2006).

[10] M. Seriu and C.-H. Wu, Phys. Rev. A 77, 022107 (2008)

[11] J.-T Hsiang, T.-H Wu, and D.-S. Lee, Phys. Rev. D 77105021 (2008)

[12] M. Brune et al., Phys. Rev. Lett. 72, 3339 (1994).

[13] M. Marrocco, M. Weidinger, R.T. Sang, and H. Walther, Phys. Rev. Lett. 81, 5784 (1998).

[14] C. I. Sukenik, M.G. Boshier, D. Cho, V. Sandoghdar, and E.A. Hinds, Phys. Rev. Lett. 70, 560 (1993).

[15] V. Druzhinina, M. DeKieviet, Phys. Rev. Lett. 91, 193202 (2003).

[16] H. G. Craighead, Science 290, 1532 (2000).

[17] Y. Sugimoto et al., Nature(London) 446, 64 (2007).

[18] M.T. Northen and K.L. Turner, Nanotechnology 16, 1159 (2005).

[19] E. M. Lifshitz, Sov. Phys. JETP, 2, 73 (1956); I. E. Dzyaloshinskii, E. M. Lifshitz and L. P. Pitaevskii, Adv. Phys. 10, 165 (1961). 
[20] M. Antezza, L. P. Pitaevskii, and S. Stringari, Phys. Rev. A 70, 053619 (2004).

[21] J. F. Babb, G. L. Klimchitskaya, and V. M. Mostepanenko, Phys. Rev. A 70, 042901 (2004);

M. Bordag, B. Geyer, G. L. Klimchitskaya, and V. M. Mostepanenko, Phys. Rev. B 74, 205431 (2006); E. V. Blagov, G. L. Klimchitskaya, and V. M. Mostepanenko, Phys. Rev. B 75, 235413 (2007).

[22] M. Antezza, L. P. Pitaevskii, and S. Stringari, Phys. Rev. Lett. 95, 113202 (2005).

[23] A. D. McLachlan, Proc. R. Soc. A 274, 80 (1963); C. Henkel et al., J. Opt. A: Pure Appl. Opt. 4, S109 (2002).

[24] T. Nakajima, P. Lambropoulos, and H. Walther, Phys. Rev. A 56, 5100 (1997); S.-T. Wu and C. Eberlein, Proc. R. Soc. A 456, 1931 (2000).

[25] M.-P. Gorza and M. Ducloy, Eur. Phys. J. D 40, 343 (2006).

[26] S. Y. Buhmann and S. Scheel, Phys. Rev. Lett. 100, 253201 (2008).

[27] T. A. Welton, Phys. Rev. 74, 1157 (1948)

[28] G. S. Agarwal, Phys. Rev. A 12, 1475 (1975), and references therein.

[29] L. Spruch and E. J. Kelsey, Phys. Rev. A 18, 845 (1978); L. Spruch, Phys. Today 39, (11), 37 (1986).

[30] C. A. Lütken and F. Ravndal, Phys. Scr. 28, 209 (1983); Phys. Rev. A 31, 2082 (1985).

[31] J. M. Wylie and J. E. Sipe, Phys. Rev. A 30, 1185 (1984); 32, 2030 (1985).

[32] A. O. Barut and J. P. Dowling, Phys. Rev. A 36, 649 (1987); 36, 2550 (1987).

[33] T. Boyer, in Foundations of Radiation Theory and Quantum Electrodynamics, edited by A. O. Barut (Plenum, New York, 1980), p. 49.

[34] P. Milonni and P. Knight, Opt. Commun. 9, 119 (1973).

[35] J. R. Ackerhalt, P. L. Knight and J. H. Eberly, Phys. Rev. Lett. 30, 456 (1973); P. W. Milonni, Phys. Rev. A 25, 1315 (1982).

[36] I. R. Senitzky, Phys. Rev. Lett. 31, 955 (1973).

[37] P. W. Milonni, J. R. Ackerhalt and W. A. Smith, Phys. Rev. Lett. 31, 958 (1973).

[38] P. W. Milonni, Phys. Rep. 25, 1 (1976).

[39] J. Dalibard, J. Dupont-Roc and C. Cohen-Tannoudji, J. Phys. (France) 43, 1617 (1982). 
[40] J. Dalibard, J. Dupont-Roc and C. Cohen-Tannoudji, J. Phys. (France) 45, 637(1984).

[41] D. Meschede, W. Jhe and E. A. Hinds, Phys. Rev. A 41, 1587 (1990).

[42] J. Audretsch and R. Müller, Phys. Rev. A 50, 1755 (1994).

[43] J. Audretsch and R. Müller, Phys. Rev. A 52, 629 (1995).

[44] J. Audretsch, R. Müller and M. Holzmann, Class. Quan. Grav. 12, 2927 (1995).

[45] R. Passante, Phys. Rev. A 57, 1590 (1998).

[46] H. Yu and S. Lu, Phys. Rev. D 72, 064022 (2005).

[47] Z. Zhu, H. Yu and S. Lu, Phys. Rev. D 73, 107501 (2006).

[48] H. Yu and Z. Zhu, Phys. Rev. D 74, 044032 (2006).

[49] H. Yu and W. Zhou, Phys. Rev. D 76, 027503 (2007).

[50] H. Yu and W. Zhou, Phys. Rev. D 76, 044023 (2007).

[51] L. Rizzuto, Phys. Rev. A 76, 062114 (2007).

[52] Z. Zhu and H. Yu, J. High Energy Phys. 02, 033 (2008).

[53] G. Compagno, R. Passante, and F. Persico, Atom-Field Interactions and Dressed Atoms (Cambridge University Press, Cambridge 1995). 


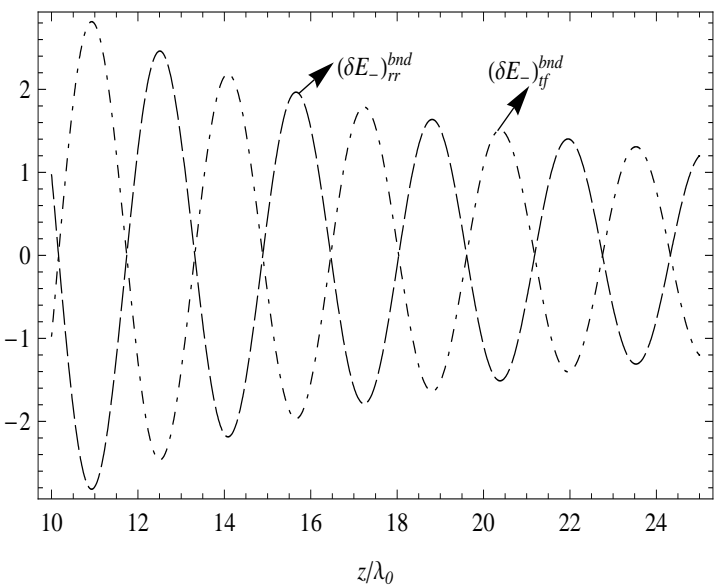

(a)

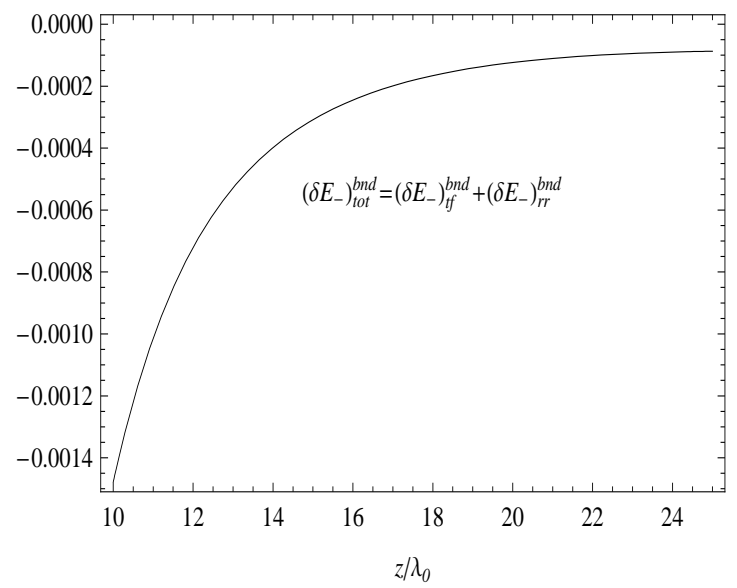

(b)

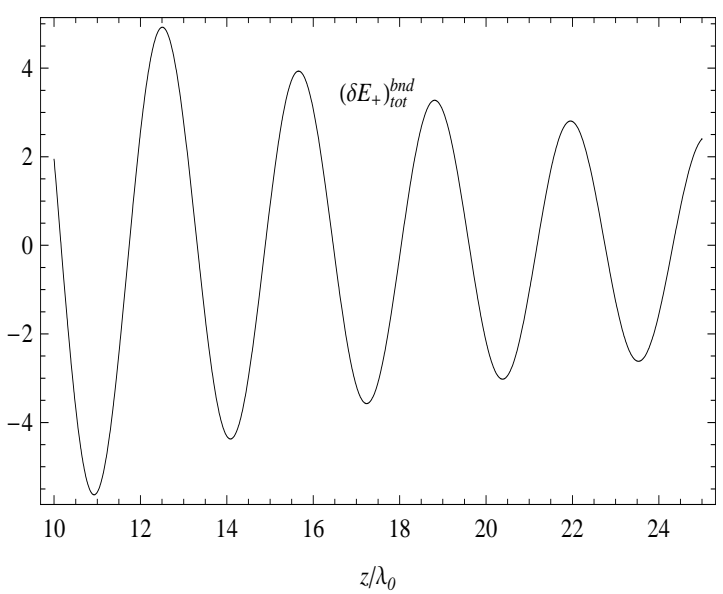

(c)

FIG. 1: Energy level shifts of both a ground state atom and an excited one polarized isotropically in the intermediate distance regime $\left(\lambda_{0} \ll z \ll \beta\right)$ at room temperature $T=300 K$. Here the frequency of $\mathrm{Rb}$ atom, $\omega_{0}=2.37 \times 10^{15} \mathrm{~s}^{-1}$ is used, and the levels shifts are in the units of $\alpha_{0} /\left(4 \pi \varepsilon_{0}\right)$. (a) The contributions of thermal fluctuations (dash-dotted line) and radiation reaction (dashed line) to the level shift of a ground state atom is plotted, demonstrating the magnitude of two effects are comparable and there is no clear domination of one over the other. (b) The level shift of a ground state atom as a net result of two competing effects is plotted. The net result is clearly much less than any of the competing effects. (c) The energy level shift of an excited state atom, Eq. (55), is plotted, showing clearly differences both in magnitude and sign from that of a ground state. 